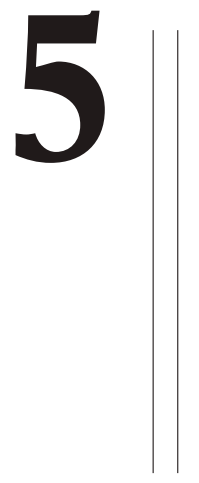

\title{
FAMILIA Y ESCUELA ANTE LA INCORPORACIÓN DE LAS TECNOLOGÍAS DE LA INFORMACIÓN Y LA COMUNICACIÓN
}

\author{
(FAMILY AND SCHOOL IN FACE OF THE INCORPORATION OF \\ INFORMATION AND COMMUNICATION TECHNOLOGIES)
}

\author{
Javier Ballesta Pagán y $\mathrm{M}^{\mathrm{a}}$ Carmen Cerezo Máiquez \\ Universidad de Murcia
}

\section{RESUMEN}

El objetivo de esta investigación ha sido conocer la valoración que hace la familia del alumnado (autóctono y extranjero) de Educación Infantil y Primaria de la Comunidad Autónoma de la Región de Murcia, sobre el uso y la interacción comunicativa con las TIC. En la metodología se utiliza un diseño transversal en consonancia con la finalidad investigadora, para recoger la opinión de la muestra seleccionada de 1932 familias, mediante la aplicación del cuestionario como instrumento de recogida de información, para explorar diferentes dimensiones referidas a: la participación, sentimientos y creencias, multiculturalidad y curricular que recoge la importancia que se le da al uso de las TIC en el proceso de enseñanza-aprendizaje, la utilización de estas tecnologías en el hogar, la valoración sobre la intencionalidad comunicativa para una mejora de la información entre la escuela y la familia, así como la formación de la familia sobre estas tecnologías.

De los resultados obtenidos se constata que las familias muestran un gran interés en el uso y en la incorporación de estos medios digitales en las aulas, a la vez que están muy interesadas en recibir formación en los propios centros educativos sobre las posibilidades de estas herramientas tecnológicas. En relación al uso de las Tecnologías de la Información y de la Comunicación en el hogar hay grandes diferencias según el país de procedencia y el nivel de estudios de los progenitores. Esta cualificación de padres y madres condiciona la valoración que se hace sobre TIC tanto en el proceso de enseñanza /aprendizaje de sus hijos, como en el uso que de éstas se desarrolla dentro del hogar. 


\section{ABSTRACT}

The main purpose of this study deals with the exploration of how the families of native and foreign pupils from certain infant and primary schools in the Autonomous Community of Murcia perceive the use and interaction with ICTs. Data were collected from 1932 families by means of a questionnaire that included the following dimensions: participation, feelings and beliefs, multiculturalism, and a number of curricular guidelines including the importance of ICTs in teaching and learning processes, the interaction between the school and the family, the use of ICTs at home and the need to train families in ICTs use. The results showed that the families were generally interested in being trained in ICTs use and in the incorporation of these digital media in the classroom. ICTs use at home varied as a function of the home country and the educational background of the pupils' parents. The qualification of parents was also found to have some bearing on how they valued ICTs in relation to their children's processes of learning and their use of these technologies at home.

\section{INTRODUCCIÓN}

La sociedad digital está configurando un modo de acceder, usar e interaccionar con las tecnologías que sobrepasa los límites de la escuela y cubre un amplio entorno socio-cultural y formativo (Bautista, 2007; Ballesta, 2009; García Areito, 2009; San Martin, 2009). Ante este panorama las principales instituciones mediadoras en la formación de los jóvenes (escuela y familia) necesitan comprender el proceso de inmersión en esa cibersociedad con el objetivo de interpretar esta realidad que condiciona el aprendizaje de los alumnos, y a la vez configura su formación personal. Sin duda, el acceso a la interacción comunicativa desde Internet ha supuesto un aumento significativo en el uso de las herramientas y en las tareas colaborativas asignadas a los medios tecnológicos que utilizamos en la formación (Sevillano, 2009). De hecho, con la incorporación de las Tecnologías de la Información y de la Comunicación (TIC) se pretende facilitar el acceso a nuevas fuentes de conocimiento, para dotar de nuevos servicios y recursos a los ciudadanos.

Sin embargo, no hay que olvidar que, al mismo tiempo, se constata una brecha digital que es una manifestación de la brecha social, donde se refleja una situación de diferencia de acceso, de desigualdad de oportunidades de participación que es entendida como la distancia entre aquellas personas y comunidades que acceden y utilizan eficazmente las tecnologías y los que no, constatándose por ello barreras y limitaciones debidas tanto por el equipamiento, como por los diferentes usos de los contenidos, en razón de la edad, sexo, desigualdades socio-culturales y entorno familiar (Bautista, 
2010; García, 2006; Tirado, 2007; Ballesta y Lozano, 2007). En este sentido, pensamos que existen brechas tecnológicas en el acceso y consumo de las tecnologías, desde la familia de origen, donde hay que profundizar en el conocimiento y valoración que tienen hacia el uso y la interacción con las TIC. Por ello, como señalan Ricoy, Feliz y Sevillano (2010) hemos de aprovechar el papel que juega la educación como llave para reducir las distancias entre los diferentes segmentos sociales y el uso de las nuevas tecnologías sabiendo que hay que facilitar el acceso y su integración en la vida social y en concreto desde la familiar. Esto ha hecho que, de forma general, bajo directrices similares, la mayoría de los países hayan llevado iniciativas en la realización de proyectos a escala nacional y regional para introducir y potenciar el uso de las tecnologías en casa, en la empresa, en universidades, institutos y escuelas. Sin olvidar que a la escuela se le exige que se implique en la socialización de las TIC y a ella llegan, con más o menos retraso, los nuevos medios tecnológicos cuyo valor sustantivo, en cuanto a lo académico y didáctico, está aún por demostrar (San Martín, 2009).

Al mismo tiempo, no todo son éxitos y seguimos cuestionando el efecto de esta incorporación de las tecnologías en los centros educativos, porque los datos recientes no son muy optimistas (Sigalés, Mominó, Meneses y Badía, 2009) y nos muestran una débil integración de las TIC, asegurando la contradicción de que docentes y alumnos son, con respecto a la sociedad, buenos usuarios de Internet, cuentan con un dominio más que aceptable y por encima de la media de los ciudadanos, pero que dos tercios de los centros no tienen un plan específico de integración de las TIC. Estos resultados desvelan que falta una cultura de utilización de las TIC que potencie nuevas comunicaciones, proyectos, relaciones e innovaciones (De Pablos, Colás y González, 2010), que van más allá de la simple utilización del ordenador. En este sentido, las distintas administraciones autonómicas, se han planteado llevar a la escuela hacia el futuro de la mano de las TIC, los programas escuela 2.0 quieren con la introducción de ordenadores portátiles en las aulas en determinados cursos realizar un avance basado en la escuela del mañana. Sin embargo, cambiar las concepciones y las formas de hacer de la escuela y los modelos de enseñar del profesorado, para acercar la acción docente a lo que se propone como constitutivo de la escuela del futuro, requiere mucho más que contar con ordenadores (Sancho, 2009; Peirats, Sales y San Martín, 2009). De hecho, según Area (2010), el uso de los ordenadores y demás tecnologías digitales no se traduce en un replanteamiento significativo y radical del modelo didáctico empleado porque, entre otros motivos, el profesorado no está completamente preparado para integrar las TIC en su práctica habitual de aula (Valverde, Garrido y Sosa, 2010). De ahí que para mejorar el modelo didáctico que garantice una integración de las TIC en los procesos de enseñanza-aprendizaje habrá que avanzar en la relación con propuestas donde colabore toda la comunidad educativa. En este sentido, las TIC deben 
ir relacionadas con la formación que se desarrolla en los centros educativos e intentar avanzar en modelos integradores que vayan entrelazando y cuestionando aquellas prácticas que sirvan para formar más allá de ser técnicas o estrategias facilitadoras de actividades empaquetadoras de determinados objetivos. Una integración efectiva de ellas en los contenidos y en los aprendizajes exige una atención aún más atenta a su presencia en la formación del profesorado (Escudero, 2009) y en establecer contextos formativos donde la familia pueda participar, así como colaborar en este proceso integrador que se está desarrollando.

Por ello, pensamos que al abordar la integración de las TIC en los centros educativos es necesaria, además, contar con la implicación de la familia y de su colaboración. Desde esta actuación, en nuestro caso, implicará abordar la dimensión formativa y comunicativa entre las TIC y la comunidad educativa, para establecer un mayor conocimiento sobre qué y cómo estas tecnologías pueden ser instrumentos que faciliten los aprendizajes entre los usuarios y mejoren las posibilidades formativas del alumnado.

En este sentido sabemos que es el hogar del joven, desde donde se establece el mayor tiempo de interacción con las TIC, aunque el modelo comunicativo que se establece viene caracterizado por ser individual y personal, en función de su propia elección, intereses y formación, al igual que no está tan claro que sea el interés informativo o formativo el que prevalezca (Rubio, 2009). Esta realidad implica como señalan Graner et al (2007), que la inmensa mayoría de jóvenes y adolescentes disponen de Internet en sus hogares y se conectan, en un porcentaje cercano al $82 \%$ de manera diaria por motivos de índole académica o relacionados con aspectos lúdicos o de ocio y contando para ello con un complejo aparataje tecnológico facilitado desde el hogar. Así, de este modo, un 65\% de niños y jóvenes entre 10 y 15 años disponen de teléfono móvil, un 40,4\% tiene televisión en su dormitorio, un $40 \%$ dispone de reproductores de música y un 57,4\% tiene consola de videojuegos (Lara et al, 2009). El uso y consumo de las nuevas tecnologías especialmente está asociado al ocio, el entretenimiento, pues sirven para oír música, ver películas, chatear, jugar y estar comunicado con el resto de compañeros, todo ello sin salir de la propia habitación. Este perfil de consumidor joven que se desarrolla fundamentalmente desde el hogar, es el que se imita y sirve de modelo al alumnado de menor edad. Del mismo modo, las diferencias entre jóvenes y adultos se constatan, en cuanto al consumo de medios, los primeros buscan diversión, entretenimiento y relación, los segundos más información (Medrano, Airbe y Palacios 2010).

De este modo pensamos que la investigación que hemos realizado sobre la valoración que realizan las familias de los alumnos, en su conjunto, nos puede ayudar a explorar algunas percepciones que se plantean hoy en 
relación a cómo las TIC pueden ser instrumentos de utilidad en la formación de los escolares. Asimismo, estamos interesados en analizar las situaciones de exclusión y desigualdad que ese están produciendo, por los efectos de las TIC en la sociedad y en los hogares del alumnado, en función de determinadas diferencias (formativas, culturales, económicas, procedencia extranjera). Estamos convencidos que el acceso a los ordenadores y a Internet por parte de la comunidad educativa, requiere de actuaciones conjuntas que faciliten y garanticen un uso eficaz y social de las tecnologías.

\section{OBJETIVOS}

Con esta investigación se pretende responder a la valoración que tienen las familias ante la incorporación de las TIC.

El objetivo general ha sido conocer la opinión que tienen los progenitores sobre el uso y la interacción comunicativa con las TIC. En concreto los objetivos específicos planteados han sido:

a) Conocer la importancia que se le da al uso de las TIC en el proceso de enseñanza-aprendizaje del alumnado.

b) Valorar la utilización de estas tecnologías en el hogar.

c) Conocer la intencionalidad comunicativa de las TIC para una mejora de la información entre la escuela y la familia.

d) Valorar la formación de la familia sobre las TIC.

\section{METODOLOGÍA}

\subsection{Población y muestra}

La población objetivo queda definida como las familias del alumnado escolarizado durante el curso 2005/06 en la etapa de la Educación Infantil y Primaria en Centros de la Región de Murcia. Para la selección de la muestra hemos utilizado un procedimiento aleatorio, en particular, un muestreo aleatorio por conglomerados y etapas (polietápico).

La muestra productora de datos la componen 1932 familias de 15 centros de Educación Infantil y Primaria de la Región de Murcia. De estas familias 1403 son autóctonas, que representa el 72,61\% de la muestra total y 529 familias extranjeras con un 27,39\%. 
El índice de confianza de la muestra es de un $95 \% \pm 2.3$ de error muestral, lo que representa que el instrumento es significativo y se puede extender al universo muestral que en este caso son 148740 alumnos de Educación Infantil y Primaria de la Región de Murcia.

\subsection{Diseño}

Se ha utilizado un diseño transversal en consonancia con la finalidad investigadora siendo el más utilizado en la investigación por encuesta. Se ha elegido este método en la medida que nos permite obtener información representativa con poblaciones diferentes en segmentos y estratos varios en contextos socioeducativos complejos. De este modo se ha recogido información de una muestra representativa en un determinado momento puntual con la finalidad de obtener una información referida a aspectos muy diversos relacionados con los objetivos planteados.

\subsection{Instrumento}

El instrumento de recogida de información ha sido un cuestionario realizado a las familias en base a diferentes aspectos exploratorios, entre las que se encontraban: participación y relaciones; sentimientos y creencias; curricular y multicultural. Ha sido en la dimensión curricular, desde donde hemos planteado un conjunto de cuestiones referidas a conocer la percepción y valoración de la familia sobre el uso de las TIC en la escuela, en el hogar y la comunicación con el centro, mediante el ordenador, así como la formación.

Para la aplicación de los cuestionarios se realizaron una serie de fases:

a) Contacto con la Administración Educativa para solicitar su colaboración en el desarrollo de la investigación facilitando datos sobre los centros educativos que escolarizan al alumnado extranjero.

b) En función de los datos proporcionados por la Consejería de Educación, Formación y Empleo se efectuó una selección de aquellos centros educativos de la Región de Murcia que escolarizan alumnado extranjero utilizando la técnica de muestreo no probabilístico.

c) Una vez realizada esta selección de centros educativos se realizó un contacto telefónico con un representante del equipo directivo de cada uno de los centros para explicar el proyecto y los objetivos del mismo, así como el modo de participación en la recogida de información. Al mismo tiempo se 
les solicitó permiso a ellos y a su claustro para desarrollar la investigación en su centro.

d) Posteriormente, se envió a cada centro el número de cuestionarios que se concretó previamente, a través del contacto establecido.

Una vez entregado los cuestionarios en el centro se realizó un compromiso con cada uno, a través de la persona de contacto, de modo que el equipo directivo del centro o quien ellos considerasen oportuno, se encargaría de la distribución a cada docente-tutor, para que los repartiese a sus alumnos, ya que los alumnos y alumnas serían intermediarios entre la escuela y la familia, teniendo en cuenta que aunque la familia tuviese varios hijos, sólo se le entregaría un ejemplar. En aquellos centros donde las familias no dominasen el castellano, se buscaría el modo de que algún miembro de la misma que lo conociese, otra familia o bien algún intérprete pudiese ayudar en dicho proceso. Para la devolución de los mismos se les dio un margen de tiempo suficiente para que el mayor número de familias hicieran su entrega. Una vez cumplimentados los cuestionarios por parte de las familias y avisados por los centros que ya disponíamos de ellos, se recogieron todos los cuestionarios para su vaciado y posterior análisis.

\subsection{Proceso-procedimiento de análisis de datos}

La información recogida de los cuestionarios dirigidos a las familias ha sido tratada mediante técnicas estadísticas de carácter descriptivo. Para tales análisis cuantitativos hemos utilizado el programa Statistical Package for the Social Sciences (SPSS 15.0).

El procedimiento seguido a la hora de la recogida de datos es el que a continuación relatamos:

a) Recoger de los centros los cuestionarios.

b) Realizar la clasificación de cuestionarios por centros y numerarlos, para realizar el posterior análisis estadístico.

c) Codificación y tratamiento estadístico de los datos. anómalos)

d) Estudio exploratorio de datos (depuración y corrección de datos

e) Técnicas estadísticas para variables cualitativas o categóricas (cálculo de frecuencias y porcentajes) y elaboración de gráficos exploratorios. 
En primer lugar se realiza un análisis descriptivo general sobre la muestra total, para, en un segundo momento, realizarlo en función de quién respondió a las cuestiones formuladas (padre-madre, padre, madre y otros); en segundo lugar, se hace el análisis según la procedencia de la familia (autóctona o extranjera); en tercer lugar, según el país de origen agrupados en diferentes categorías (España, UE, Este, Latinoamérica, Árabe, Oriente, otros) y por último se analizan los datos atendiendo al nivel de estudios de los progenitores (sin estudios, básicos, medios y universitarios).

\section{RESULTADOS}

En relación a la primera cuestión analizada sobre la valoración que realizaban las familias en relación al uso de las TIC en el proceso de enseñanzaaprendizaje los resultados nos confirman que el 85,8\% de las familias opinan que es bastante importante el uso de las TIC y tan sólo el 3,8\% consideran es nada o poco interesante esta utilización, como se aprecia en el gráfico 1

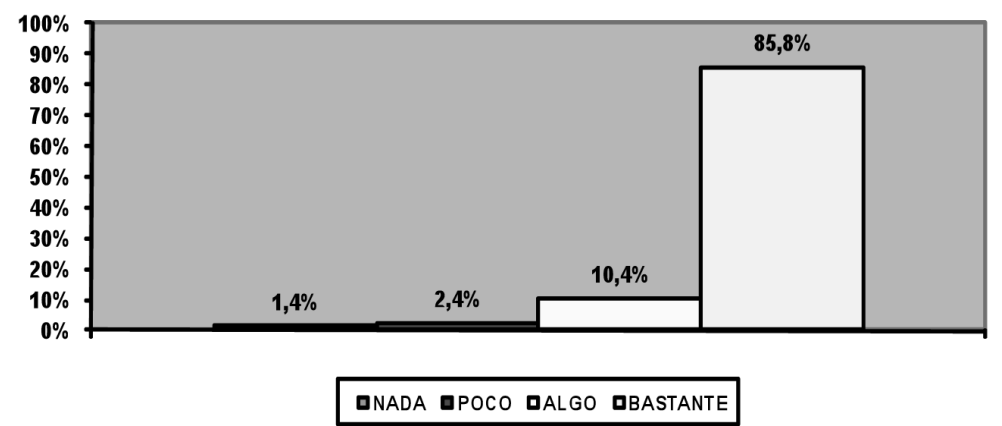

Gráfico1. Importancia de las TIC en el Proceso de Enseñanza- Aprendizaje

No obstante, sobre esta utilización de las TIC en casa, el 68,7\% lo consideran muy importante, y casi un tercio no lo utilizan. 


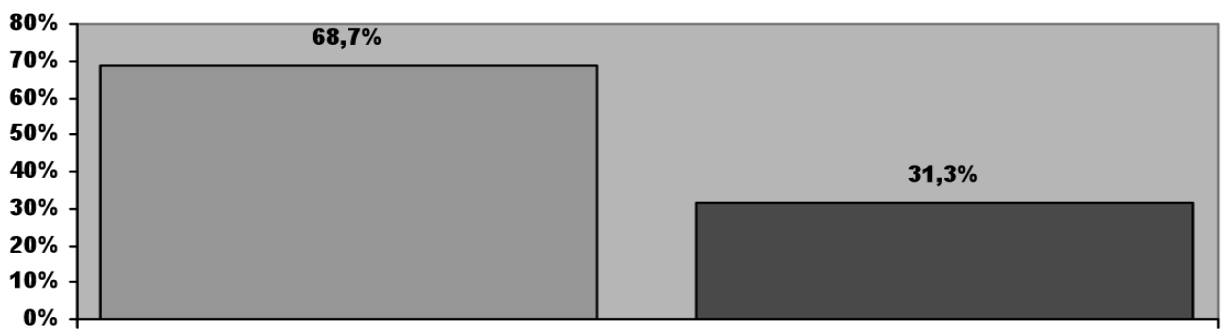

$\square \mathrm{SI} \square \mathrm{NO}$

Gráfico 2. Utilización de las TIC en casa

Un $69,3 \%$ de las familias encuestadas consideran que les gustaría recibir o dar información del centro y/o de su hijo a través del ordenador desde sus casas, un 30,7\% no están de acuerdo con esta afirmación.

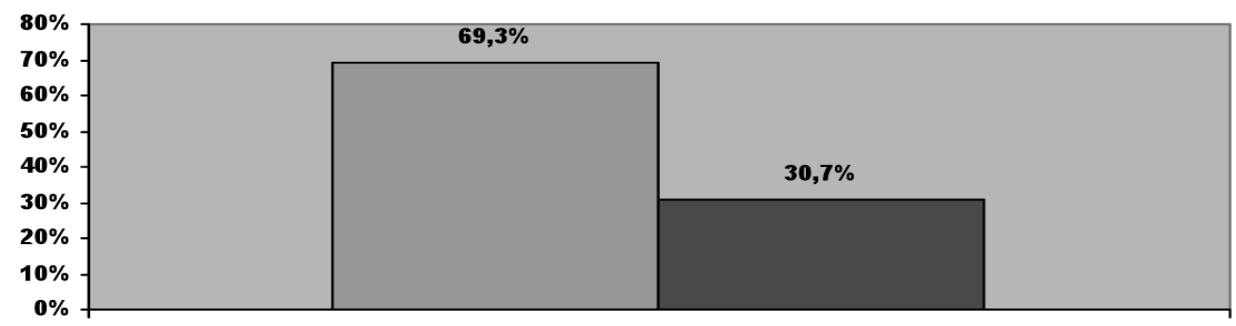

$$
\square \mathrm{SI} \text { aNO }
$$

Gráfico 3. Recibir o dar información del centro o de su hijo a través del ordenador desde su casa

Casi la totalidad de las familias $(88,7 \%)$ opinan favorablemente sobre recibir formación en el centro sobre el uso de las TIC.

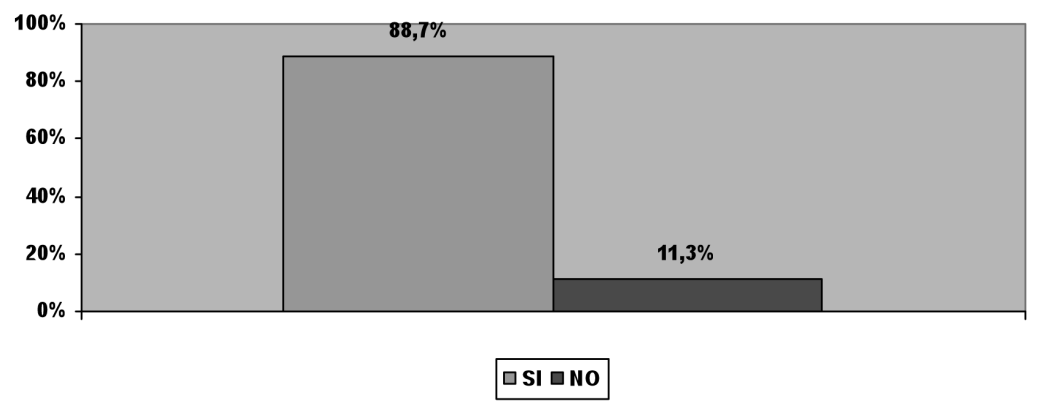

Gráfico 4. Recibir formación en el centro sobre uso de TIC 


\subsection{Atendiendo a quién responde al cuestionario}

Para dar respuesta a esta cuestión le dimos 4 opciones que lo hicieran de forma conjunta el padre y la madre, el padre solamente, o la madre u otro familiar (esta opción la marcamos principalmente pensada en las familias de origen extranjero que, a lo mejor, no dominaban el castellano y necesitaban la ayuda de un hijo mayor, u otro familiar con mayor competencia lingüística, etc.)

Tal y como se puede observar en el gráfico siguiente no importa quién de respuesta, ya que los cuatro grupos están bastante de acuerdo en la importancia del uso de las tecnologías en el proceso de E/A de su hijos, en unos porcentajes bastantes similares, alrededor de un $80 \%$. Solamente hay un dato que habría que destacar en el grupo de "otros", un 14,6\% opinan que nada o poco le importa el uso de las TIC, frente al resto de los grupos que ninguno de ellos supera el 4\%, uniendo estas dos opciones de respuesta.

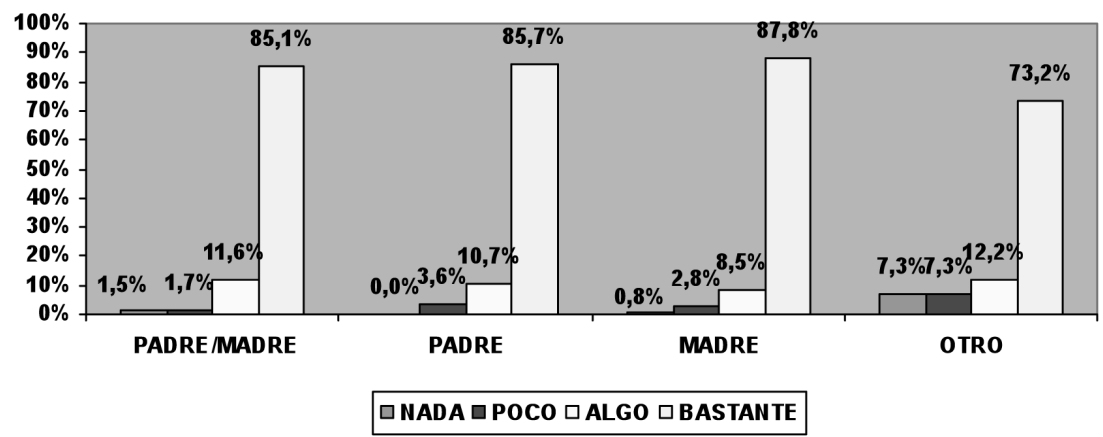

Gráfico 5. Importancia de las TIC en el Proceso de Enseñanza- Aprendizaje según quién de respuesta a la encuesta

En relación al uso que les dan a las TIC en casa atendiendo a quién responde, los datos que se encuentran son los siguientes. Las madres mayoritariamente en un 70,8\% son las que más valoran el uso que le dan a las tecnologías dentro de la casa, un porcentaje muy cercano se encuentra el grupo de padre y madre conjuntamente en un $68,8 \%$, frente al grupo de los otros que es menos de la mitad (46,3\%). En los grupos "padre/madre, padre, madre" aproximadamente en un tercio opinan que no usan las TIC dentro de casa, frente al grupo de "otros" que la mitad de los encuestados $(53,7 \%)$ no las usan dentro del domicilio familiar. 


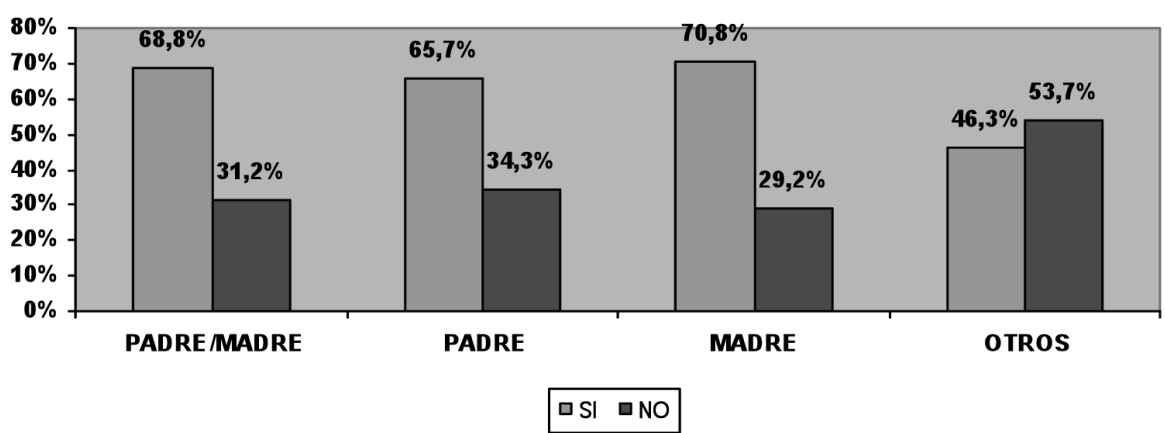

Gráfico 6. Uso de las TIC en casa según quién de respuesta a la encuesta

El siguiente aspecto a valorar hace referencia al hecho de recibir o dar información del centro y/o de su hijo a través del ordenador desde su casa. Todos los grupos en unos porcentajes bastante similares (alrededor de un $70 \%$ ) opinan favorablemente sobre el hecho de recibir o dar información del centro o de su hijo a través del ordenador o cualquier otro medio. Sin embargo, queda aproximadamente un 30\% que no están de acuerdo con este sistema de transmisión de la información en todos los grupos encuestados.

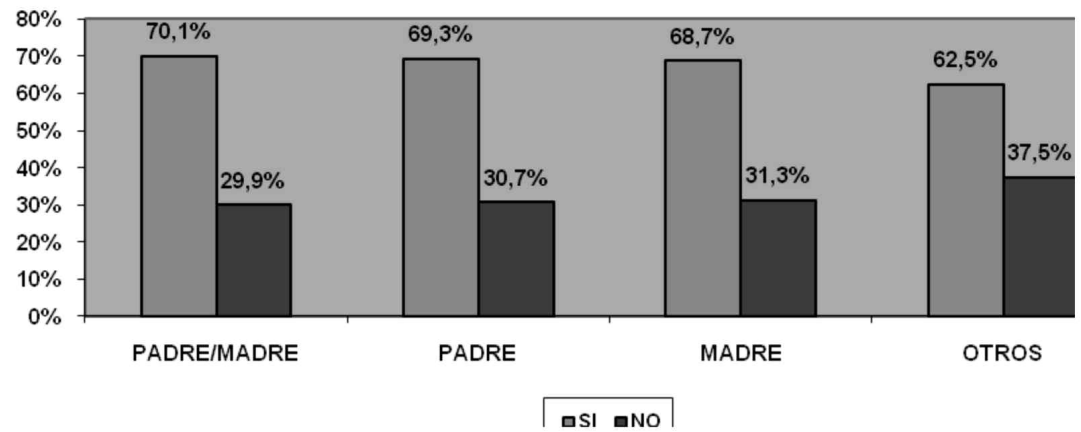

Gráfico 7. Recibir o dar información del centro y/o de su hijo a través del ordenador según quién de respuesta a la encuesta

Sobre el hecho de recibir formación en el centro educativo sobre las TIC es bien acogido por todos los grupos en unos porcentajes bastantes elevados, casi un 90\%. Solamente habría que destacar que en el grupo de "otros" hay un 19,5\% que no quiere recibir esa formación. 


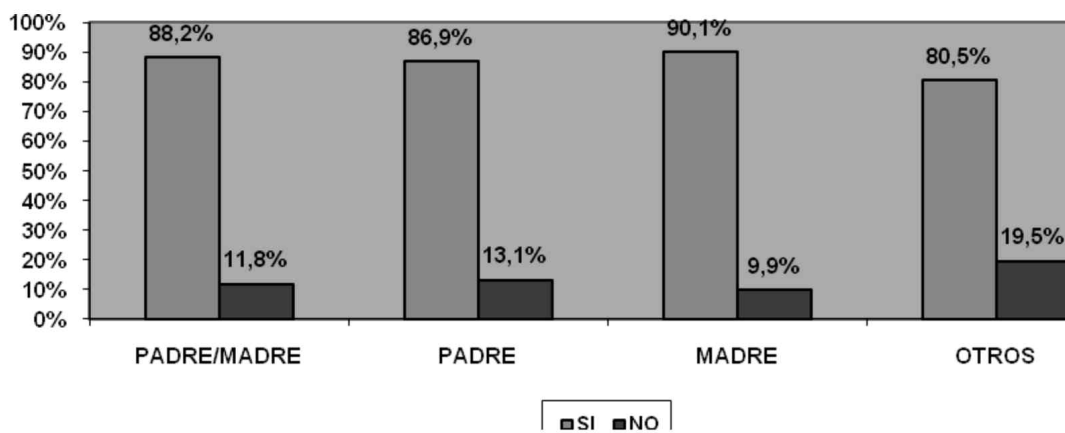

Gráfico 8. Recibir formación en el centro sobre las TIC según quién de respuesta a la encuesta

\subsection{Procedencia de la familia (autóctona-extranjera)}

Sobre la importancia de las TIC en el proceso de E/A de sus hijos, observamos que tanto las familias extranjeras como las autóctonas le otorgan bastante importancia al uso de los medios tecnológicos, en unos porcentajes similares $(82,1 \%$ y un $87,2 \%$ respectivamente), frente a estos datos habría que destacar que las familias extranjeras le dan menos importancia a este aspecto $(5,7 \%)$ que las autóctonas $(2,9 \%)$.

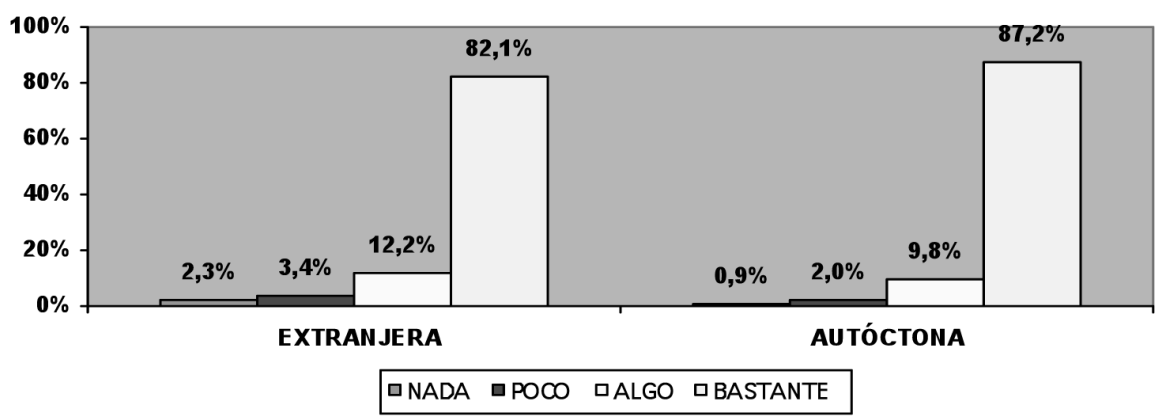

Gráfico 9. Importancia de las TIC en el Proceso de Enseñanza- Aprendizaje según la procedencia de la familia

En relación a la utilización de las TIC dentro del hogar observamos que existen grandes diferencias entre ambos colectivos, frente a las familias extranjeras que se reparten las opiniones en un 50\%, las familias autóctonas un 75\% opinan favorablemente sobre el uso de las TIC y tan sólo un cuarto de ellas no la utilizan. 


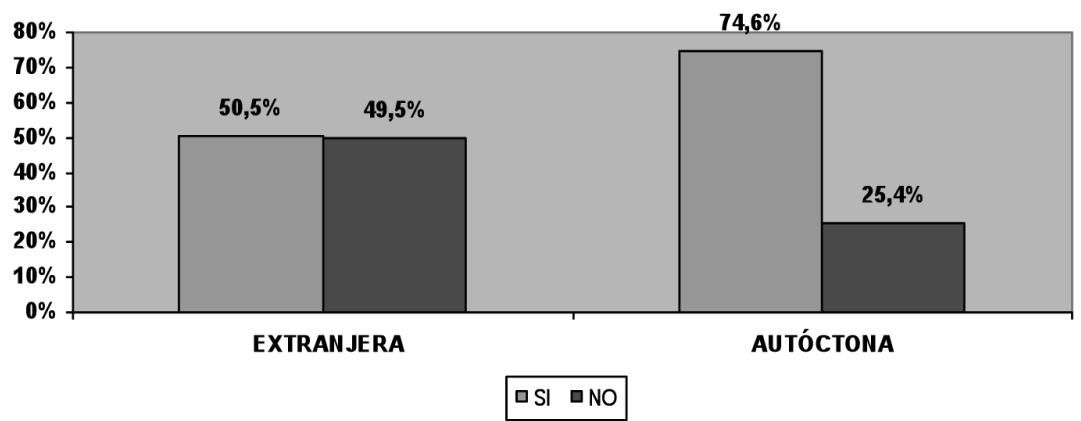

Gráfico 10. Uso de las TIC en casa según la procedencia de la familia

En el siguiente gráfico se analiza la importancia que reconocen las familias extranjeras y autóctonas sobre recibir o dar información del centro y/o su hijo a través del ordenador desde su casa. Observamos que tres cuartas partes de las familias extranjeras están a favor de utilizar las TIC para este fin frente al $67,2 \%$ de las familias autóctonas.

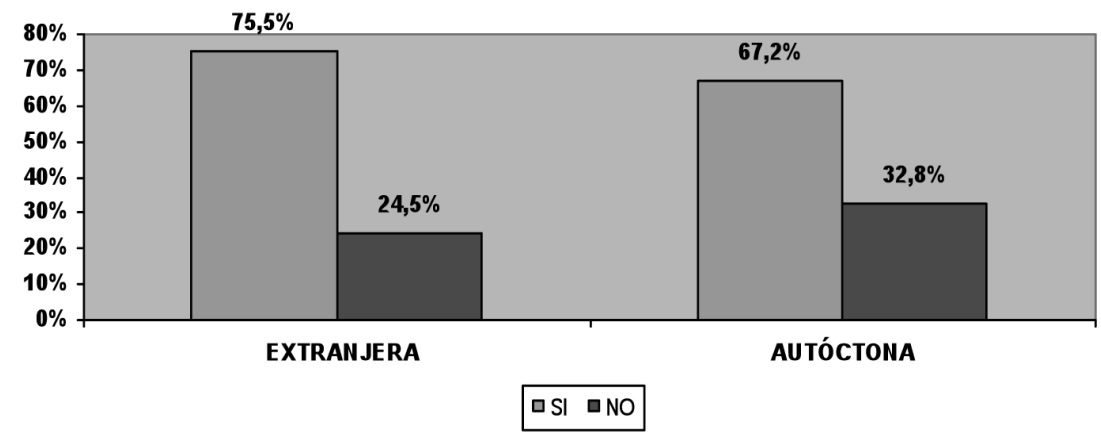

Gráfico 11. Recibir o dar información del centro y/o de su hijo a través del ordenador según la procedencia de la familia

En relación con la última dimensión, recibir formación sobre las TIC en el centro educativo, observamos que no hay diferencias entre ambos colectivos, ya que ambos en unos porcentajes muy elevados, casi el $90 \%$, piensan que les resultaría muy interesante recibir tal formación. 


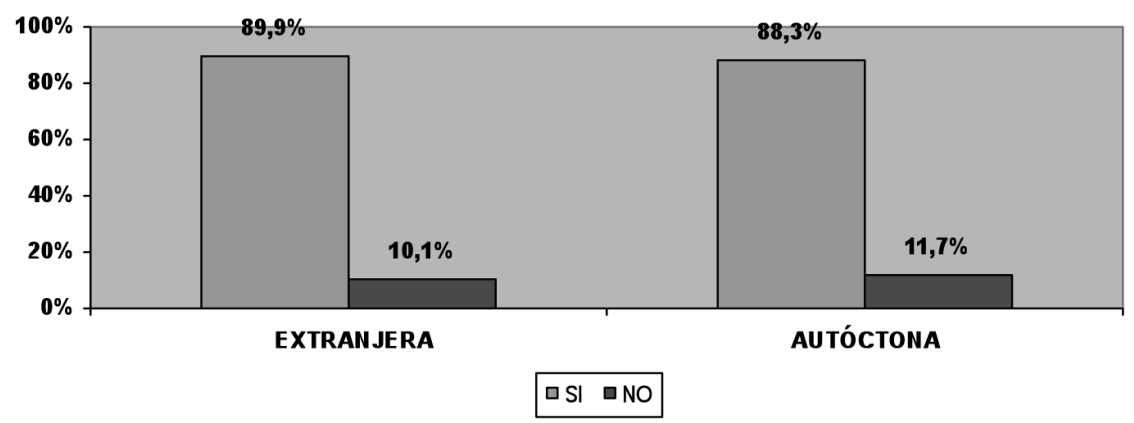

Gráfico12. Recibir formación en el centro sobre las TIC, según la procedencia de la familia

\subsection{País de procedencia}

Atendiendo al país de procedencia de la familia, hemos establecido unas categorías que anteriormente hemos descrito.

Al analizar el gráfico observamos que de los diferentes grupos de países que se ha repartido la muestra son los de la cultura árabe los que menos importancia le conceden a la incorporación de las TIC al proceso de E/A de sus hijos, siendo en los demás grupos un aspecto muy importante, según nos muestran los datos.

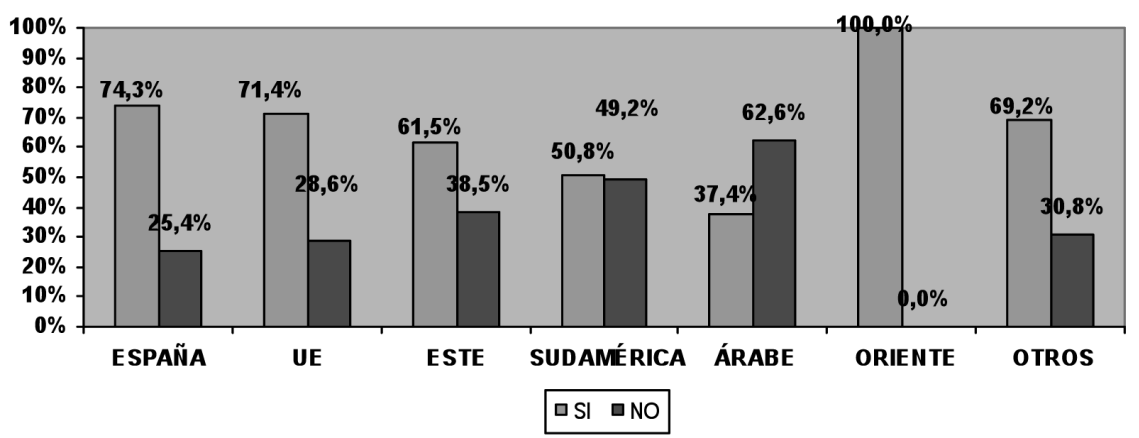

Gráfico 13. Importancia de las TIC en el proceso de enseñanza- aprendizaje según el país de procedencia de la familia

En relación con la importancia de las TIC en casa todos los grupos consideran importante su uso, en unos valores parecidos. Sin embargo, son las familias árabes las que le conceden una baja importancia, solamente un $37,4 \%$. En el resto, todos los valores rondan sobre un $70 \%$ aproximadamente. 
Habría que señalar que las familias de origen latinoamericano son las que tiene unos valores parecidos en ambas opciones de respuesta, aproximadamente la mitad de las familias lo consideran positiva la incorporación y uso de las TIC en casa frente a la otra mitad que dicen que no.

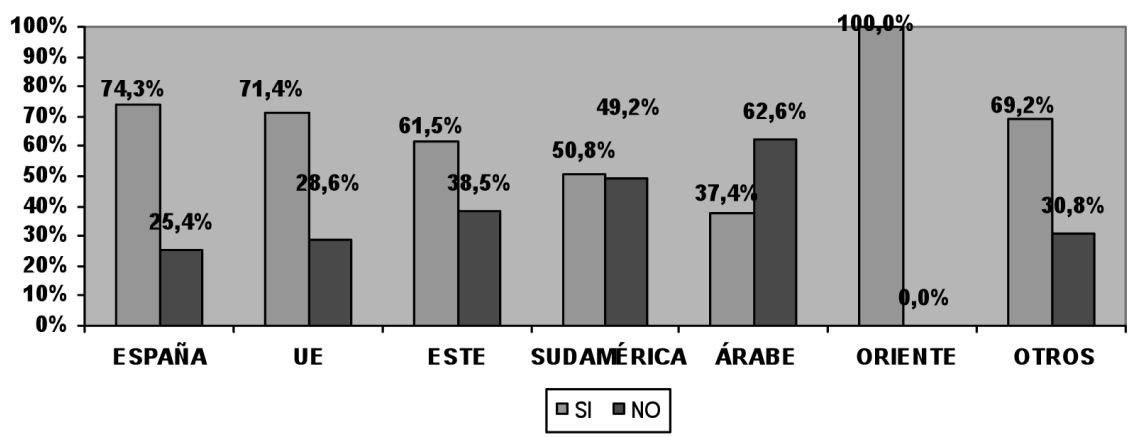

Gráfico 14. Uso de las TIC en casa según el país de procedencia de la familia

Como se observa en el gráfico siguiente todos los grupos están interesados por dar y recibir información del centro y de sus hijos a través del ordenador, sobre todo las familias de la Unión Europea, y de Europa del Este. Aunque también se observa que hay casi un tercio de familias de origen español y árabe que se encuentran reticentes ante esta situación.

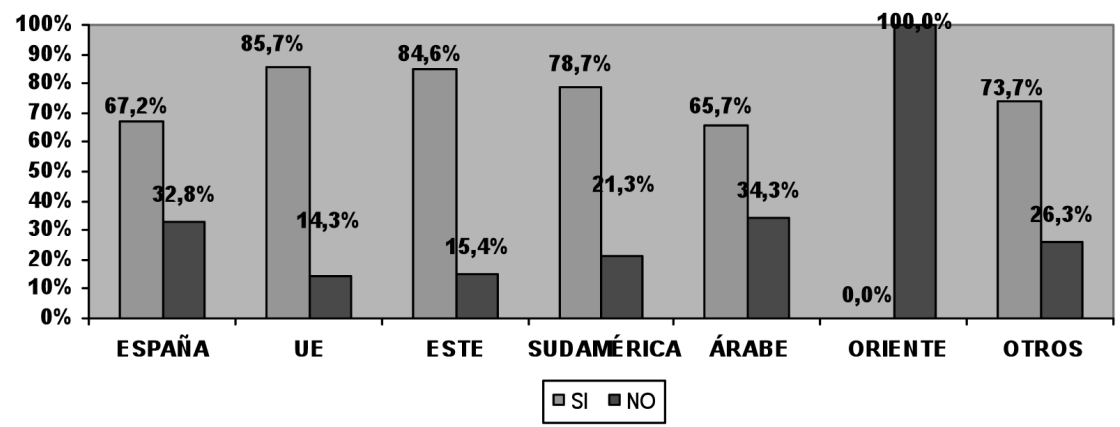

Gráfico 15. Recibir o dar información del centro y/o de su hijo a través del ordenador según el país de procedencia de la familia

Al valorar el interés por recibir formación sobre TIC en la escuela, observamos que hay un gran interés manifestado tanto por los autóctonos como por los extranjeros. Se constata que las familias están muy interesadas en recibir formación en los centros sobre el uso de las tecnologías. 


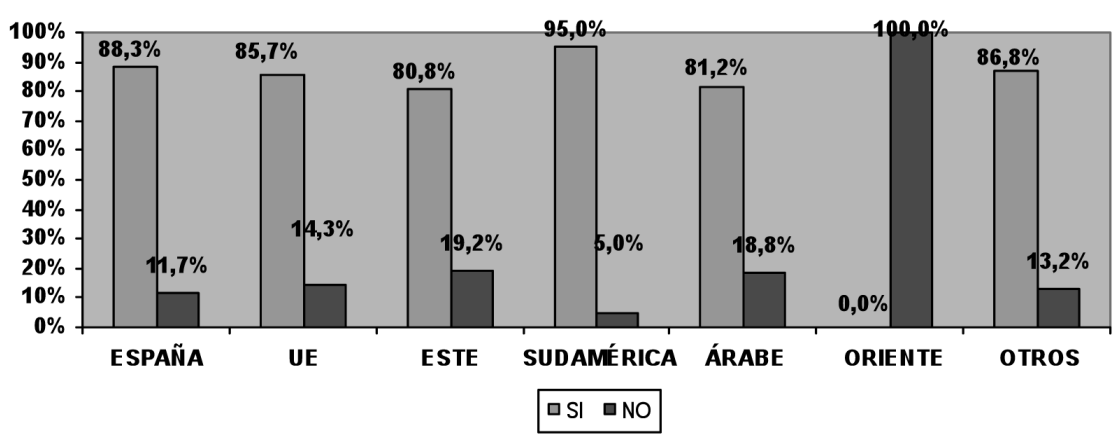

Gráfico 16. Recibir formación en el centro sobre las TIC según el país de procedencia de la familia

\subsection{Nivel de estudios del padre}

En el gráfico siguiente vemos la relación existente entre el nivel de estudios del padre y si esto influye en la importancia que le dan a las TIC, dentro del proceso de enseñanza-aprendizaje de su hijos. Observamos que los padres con niveles de estudios más altos son los que más sensibilizados están con la incorporación de las tecnologías, como era de esperar, en el proceso de E/A de sus hijos. Por otro lado habría que destacar que los "padres sin estudios" son los que consideran menos importante $(12,2 \%)$ tal incorporación al sistema educativo actual, en contraste con los universitarios que tan sólo 1,8\% no la consideran.

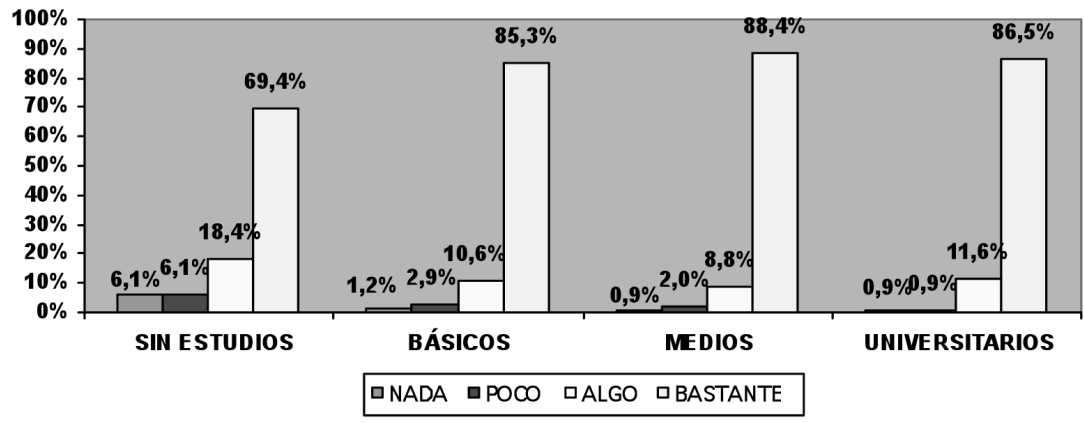

Gráfico 17. Importancia de las TIC en el proceso de enseñanza- aprendizaje según el nivel de estudios del padre

Son los "padres sin estudios" los que no usan las TIC en casa en un $64,3 \%$, frente a los padres con niveles de estudios más altos los que hacen un mayor uso de éstas dentro del ámbito familiar. Hay que destacar que los pa- 
dres con estudios universitarios usan las TIC en un $85,8 \%$ dentro de su hogar.

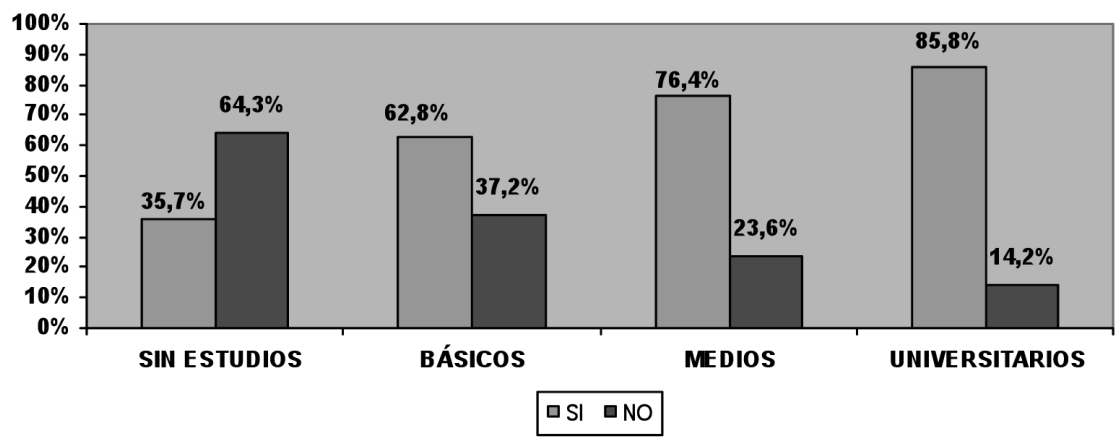

Gráfico 18. Uso de las TIC en casa según el nivel de estudios del padre

Sobre la dimensión de recibir o dar información del centro y/o de su hijo a través del ordenador, todos los padres están de acuerdo y lo consideran un medio importante de información sobre la vida de los centros educativos. Se observa que los que tienen mayores niveles de estudios consideran en un porcentaje mayor la importancia de utilizar estos medios como sistemas de comunicación con el centro.

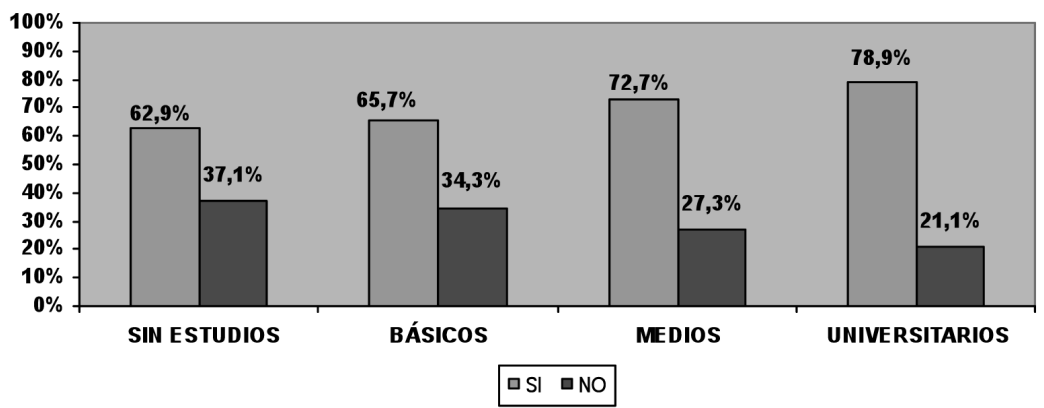

Gráfico 19. Recibir o dar información del centro y/o de su hijo a través del ordenador según el nivel de estudios del padre

Todos los padres están muy interesados en recibir formación en el centro sobre las TIC, en unos porcentajes muy elevados alrededor del 85\%. Hay que señalar que son los padres con niveles de estudios más altos y bajos los que presentan unos porcentajes menores; frente a los padres con niveles básicos o medios (90\%) quienes están más a favor de que se desarrolle este tipo de actividad en los centros educativos. 


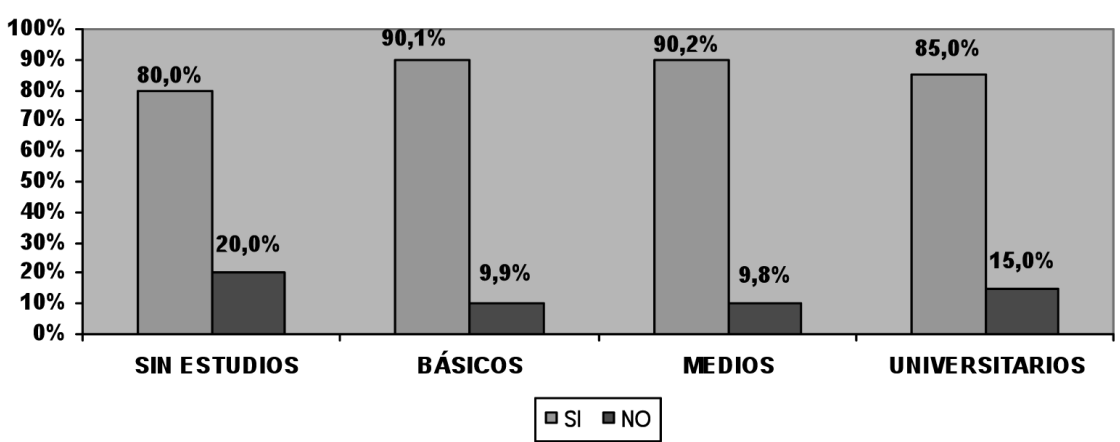

Gráfico 20. Recibir formación en el centro sobre las TIC según el nivel de estudios del padre

\subsection{Nivel de estudios de la madre}

El siguiente análisis hace referencia a la relación existente entre las dimensiones formuladas y el nivel de estudios de la madre.

Se observa que, al igual que sucedía anteriormente con los padres, las madres con mayores niveles de estudios opinan favorablemente sobre la incorporación de las TIC en el proceso de E/A de sus hijos. Del mismo modo, al igual que sucedía con los padres, son las madres con menores niveles de estudios en un porcentaje de 15,7\% las que no consideran importante la incorporación de las TIC dentro del proceso de aprendizaje de sus hijos.

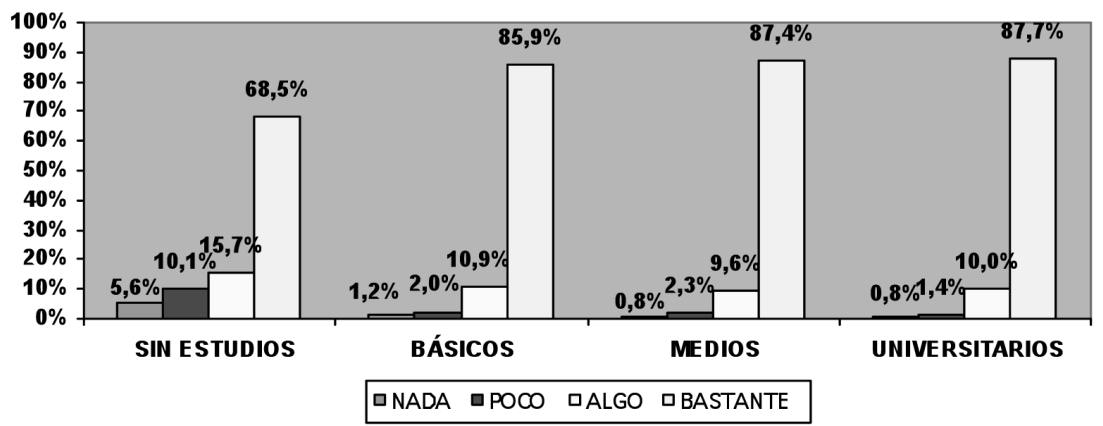

Gráfico 21. Importancia de las TIC en el proceso de enseñanza- aprendizaje según el nivel de estudios de la madre

Sobre el uso de la TIC en casa, son las madres con mayores niveles de estudio las que consideran más importante el uso de los medios dentro del hogar, tal y como sucedía con los padres. Sin embargo, las tres cuartas partes de las madres sin estudios no usan las TIC dentro del hogar. 


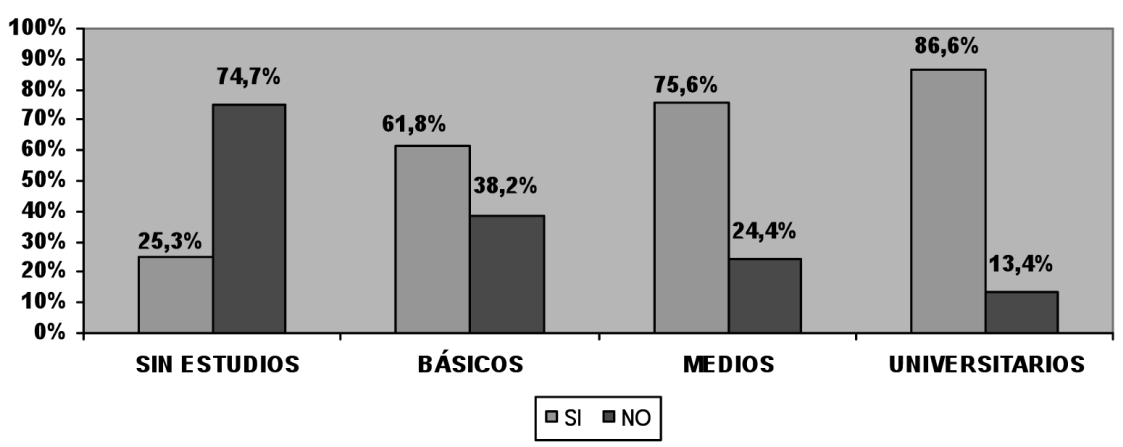

Gráfico 22. Uso de las TIC en casa según el nivel de estudios de la madre

Atendiendo a la importancia que les conceden las madres a recibir o dar información de su hijo a través del ordenador, observamos que todas están muy interesadas en usar este medio, sin embargo las madres con mayor nivel de estudios tienen unos porcentajes más elevados sobre la necesidad de información y, al mismo tiempo, son menores quienes no lo consideran de interés.

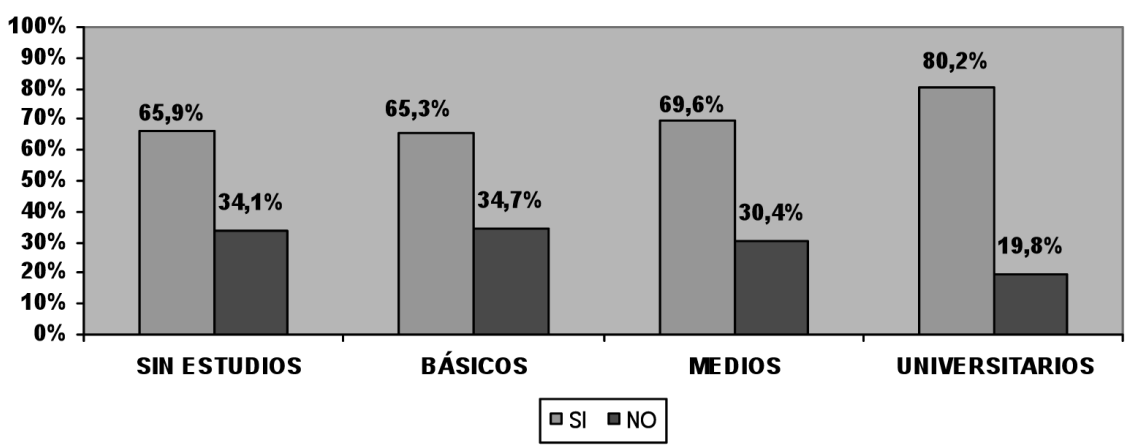

Gráfico 23. Recibir o dar información del centro y/o de su hijo a través del ordenador según el nivel de estudios de la madre

En relación a recibir formación en el centro sobre las TIC, todas las madres están muy interesadas en unos porcentajes muy elevados alrededor del $85 \%$. Hay que señalar que son las madres con niveles de estudios básicos y medios las que presentan unos porcentajes más altos (90), las que están más a favor de que se desarrolle este tipo de actividad en los centros educativos. 


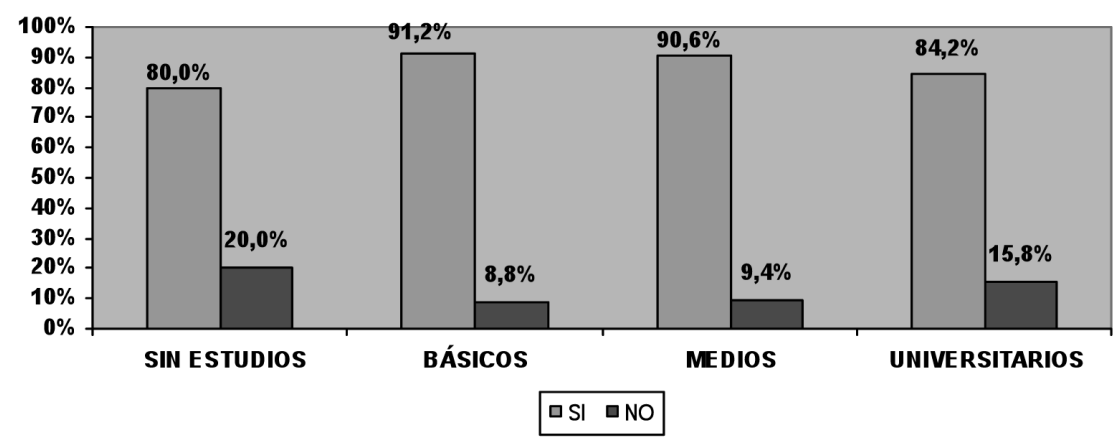

Gráfico 24. Recibir formación en el centro sobre las TIC según el nivel de estudios de la madre

\section{CONCLUSIONES}

De los resultados conseguidos en esta investigación destacamos que de todas las dimensiones analizadas, sobre las TIC y la familia en alumnos de Educación Infantil y Primaria se muestra un gran interés en el uso y en la incorporación en el proceso de enseñanza-aprendizaje. Al mismo tiempo, las familias de los alumnos están muy interesadas en recibir formación en los propios centros educativos, sobre estas herramientas tecnológicas y sus posibilidades. Este contenido formativo sería de máximo interés para ser abordado en relación a la oferta que se hace o pudiera realizarse desde los planes de actuación de las Escuelas de Padres. Estos datos nos muestran que es necesario en muchos casos abrir los diferentes contextos educativos para que los padres puedan entrar y de esta forma ser formados para el conocimiento y uso de las TIC, ya que de esta forma estaremos contribuyendo a la formación de sus hijos y de manera más genérica a la propia formación como usuarios de una sociedad digital que conlleva una alfabetización digital.

De igual modo, y en relación al uso de las Tecnologías de la Información y de la Comunicación dentro del hogar, según el país de procedencia hay diferencias, por lo que son las familias de cultura árabe y latinoamericana las que obtienen valores más bajos. Pensamos que eso es debido, principalmente, porque en sus hogares no tienen esos medios tecnológicos, para poder hacer uso de ellos debido a condicionantes económicos. Al mismo tiempo, un aspecto a tener en cuenta, es que tanto los padres como las madres dependiendo del nivel de estudios consideran importante la incorporación de las TIC tanto en el proceso de enseñanza /aprendizaje de sus hijos, como en el uso de las mismas dentro de su hogar. 
En este sentido, se puede considerar que el nivel de estudios de madres y padres va a condicionar el uso de estas tecnologías dentro del hogar familiar y este hecho puede afectar a la formación de sus hijos ante los medios tecnológicos actuales. Tal y como señalan diferentes autores nos encontramos ante una brecha digital que cada vez debe ser más corta para que no existan barreras a la hora de enfrentarnos ante las TIC, sin que el uso de las tecnologías dentro del aula y del hogar suponga una diferenciación entre el alumnado escolarizado actualmente en nuestros centros.

De lo anteriormente expuesto se constata una necesidad de profundizar en torno a esta temática, para llegar a comprender y valorar el papel que tiene la familia en el uso de las TIC y su importancia dentro del ámbito escolar, ya que las familias pueden condicionar el uso de estos medios a favor de los alumnos. Sin olvidar que hoy nos encontramos ante una sociedad digital donde toda la comunidad educativa, a nivel de usuario, necesita comunicarse a través de estas tecnologías. Este hecho supondrá que es necesario trabajar de forma coordinada familia-escuela, puesto que la actitud de ambos colectivos va a influir en el desarrollo personal y social de nuestros alumnos. 


\section{REFERENCIAS BIBLIOGRÁFICAS}

Area, M. (2010). El proceso de integración y uso pedagógico de las TIC en los centros educativos. Un estudio de casos. Revista de Educación, 352, 7797.

Ballesta, J. (Coord.). (2009). Educar para los medios en una sociedad multicultural. Barcelona: Davinci.

Ballesta, J. y Lozano, J. (2007). Los medios de comunicación, ¿nos igualan o nos diferencian? Enseñanza, 25, 45-67.

Bautista, A. (2007). Alfabetización tecnológica multimodal e intercultural. Revista de Educación, 343, 589-600.

Bautista, A. (2010). Desarrollo tecnológico y educación. Madrid: Fundamentos.

De Pablos, J., Colás, P. y González, T. (2010). Factores facilitadores de la innovación con TIC en los centros escolares. Un análisis comparativo entre diferentes políticas educativas autonómicas. Revista de Educación, 352, 2351.

Escudero, J.M. (2009). Las nuevas tecnologías y la formación del profesorado, en De Pablos, J. (ed) Tecnología Educativa. La Formación del profesorado en la era de Internet. Málaga: Aljibe, 19-25.

García Areito, L. (2009). ¿Por qué va ganando la educación a distancia? Madrid: UNED.

García, I. (2006). Políticas para la inclusión social mediante tecnologías de la información y la comunicación. Revista TIE, 20, 23-44

Graner, C., Beranuy, M., Sánchez-Carbonell, X., Chamarro, A. y Castellana, M. (2007). ¿Qué uso hacen los jóvenes y adolescentes de Internet y del móvil?
Galicia: Foro Internacional sobre Comunicación e Xuventude.

Lara, F., Fuentes, M., Fuente, R., Pérez, F., Garrote, G. y Rodríguez, M. V. (2009). Uso y abuso de las TIC en la población escolarizada burgalesa 10-18 años: relación con otras variables psicosociales. Burgos: Universidad de Burgos.

Medrano, C., Airbe, A. y Palacios, S. (2010). El perfil de consumo televisivo en adolescentes, jóvenes y adultos: implicaciones para la educación. Revista de Educación, 352, 545-566.

Peirats, J.; Sales, C. y San Martín, A. (2009). Un "portátil por estudiantes" como argumento de disputa política en la sociedad digital. Educatio Siglo XXI, 27 (2), 53-70.

Ricoy, M., Feliz, T. y Sevillano, M. L. (2010). Competencias para la utilización de las herramientas digitales en la sociedad de la información. Educación XX1, 13 (1), 199-219.

Rubio, A. (Dir.) (2009). Adolescentes y jóvenes en la red: factores de oportunidad. Madrid: Injuve.

San Martín, A. (2009). La escuela enredada. Formas de participación escolar en la Sociedad de la Información. Barcelona: Gedisa.

Sancho, J.M. (2009). ¿Qué educación, qué escuela para el futuro próximo? Educatio Siglo XXI, 27 (2), 13-32.

Sevillano, M.L. (2009). Posibilidades formativas mediante nuevos escenarios virtuales. Educatio Siglo XXI, 27 (2), 71-94.

Sigalés, C., Mominó, J. M., Meneses, J. y Badía, A. (2009). La integración de In- 
ternet en la educación escolar española. Situación actual y perspectivas de futuro. Madrid: Ariel.

Tirado, R. (2007). Las TIC en el marco de la educación compensatoria, en Cabero, J. Córdoba y Fernández, J. (eds), Las TIC para la igualdad. Sevilla: Eduforma, 173-196.
Valverde, J., Garrido, M. C. y Sosa, M. J. (2010). Políticas educativas para la integración de las TIC en Extremadura y sus efectos sobre la innovación didáctica y el proceso de enseñanza-aprendizaje: la percepción del profesorado. Revista de Educación, $352,99-124$. 


\section{PALABRAS CLAVE}

Familia, TICs, escuela, multiculturalismo, currículo

\section{KEYWORDS}

Family, ICTs, school, multiculturalism, curricula.

\section{PERFIL ACADÉMICO Y PROFESIONAL DE LOS AUTORES}

Javier Ballesta Pagán, Profesor Titular de Universidad en el Departamento de Didáctica y Organización Escolar de la Universidad de Murcia. Desarrolla su actividad investigadora en Tecnología Educativa donde viene colaborando en la realización de proyectos de investigación sobre comunicación educativa, consumo de medios digitales y formación en TIC.

$M^{a}$ Carmen Cerezo Máiquez, Maestra de Educación Primaria en el Ceip. Ricardo Campillo-Santomera (Murcia). Licenciada en Pedagogía y Psicopedagogía por la Universidad de Murcia.

Dirección autores: Javier Ballesta Pagán

$\mathrm{M}^{\mathrm{a}}$ Carmen Cerezo Máiquez

Universidad de Murcia

Facultad de Educación

Departamento de Didáctica y Organización Escolar

Campus de Espinardo 30100

Tf. 868884019

E-mail:pagan@um.es.

Fecha Recepción del Artículo: 27. septiembre. 2010

Fecha Modificación Artículo: 28. enero. 2011

Fecha Aceptación del Artículo: 10. marzo. 2011 